\title{
RESPONSE OF Ficus binnendykii (Miq.) Miq. cv. "AMSTEL QUEEN" TRANSPLANTS TO SOME BIOFERTILIZERS
}

\author{
El-Tayeb $^{(1)}$, H.F $\quad$ Boshra A. El-Sayed ${ }^{(2)}$
}

Botanical Gars. Res. Dept., Antoniades Res. Branch, Hort. Res. Inst., Alexandria, Egypt.

Ornamental Res. Dept., Hort. Res. Inst., Giza, Egypt.

\begin{abstract}
This investigation was carried out in the open field at the nursery of Antoniades Res. Branch, Hort. Res. Inst., Alexandria, Egypt during 2009 and 2010 seasons to study the effect of soil dressing with rhizobacterien, nitrobien and biogien commercial biofertilizers on growth and chemical composition of 6months-old transplants of Ficus binnendykii (Miq.) Miq. cv. "Amstel Queen" cultivated in 20 -cm-diameter clay pots filled with about $2.5 \mathrm{~kg}$ of sand + clay mixture $(1: 1, \mathrm{v} / \mathrm{v})$, when added at the rates of 0,3 and $6 \mathrm{~g} / \mathrm{pot}$, five times with one month interval, and to detect how far these biofertilizers can replace the chemical fertilization with NPK compound fertilizer at the rate of $2 \mathrm{~g} /$ pot. The obtained results indicated that all fertilization treatments examined in this study significantly improved all vegetative and root growth parameters (plant height $(\mathrm{cm})$, stem diameter $(\mathrm{mm})$, No. branches and leaves/plant, leaf area $\left(\mathrm{cm}^{2}\right)$ and fresh and dry weights (g) of leaves, stem and roots), as well as the active constituents in the leaves of the treated plants (chlorophyll a, b and carotenoids (mg/g F.W.) and the percentages of total carbohydrates, $\mathrm{N}, \mathrm{P}$ and $\mathrm{K}$ ). the dominance in all previous measurments was for the dressing with biogien at $6 \mathrm{~g} /$ pot treatment, which gave the utmost high means compared to those of control, NPK and all other biofertilization treatments in the two seasons. It was also noticed that biofertilizers treatments, especially at the high rate $(6 \mathrm{~g} / \mathrm{pot})$ recorded better results than NPK treatment in most cases of both seasons. So, in order to get the best growth and higher content of active constituents of 6-months-old transplants of $F$. binnendykii cv. "Amstel Queen" grown in 20-cm-diameter clay pots, it is preferable to fertilize them with biogien at the rate of $6 \mathrm{~g} / \mathrm{pot}$, proved to added as soil dressing, five times with one month interval.
\end{abstract}

\section{INTRODUCTION}

Ficus binnendykii (Miq.) Miq. cv. "Amstel Queen", Narrow leaf fig, that belongs Fam. Moraceae is a glabrous shrub or tree up to 6-8 $\mathrm{m}$ high, with dense branches full of green narrow leaves. Native to SE Asia to Java, Sulawesi and the Philippines. It is widely used as lawn specimens due to its huge and attractive vegetative growth, and as pot-plant in great container for decoration of sunny places. Propagated by cuttings (Huxley et al., 1992). Nowadays, using of biofertilizers, as natural preparations containing one or more of beneficial microorganisms that can release nutrients from rocks and organic matter in the soil to become available for plants, has become one of the most important requirements to protect environments from pollution, besides getting a safe and clean product. Some of microorganisms can fix atmospheric nitrogen in a free living state, 
e.g. Azotobacter and Azospirillum (Darwish, 2002). Moreover, Azotobacter bacteria secrete some growth promoting factors, e.g. gibberellin, cytokinin-like substances, auxins, as well as some vitamins such as thiamine, riboflavin, pyridoxine, nicotinic and pantothenic acids (Darwish, 2002). Subba Rao (1993) indicated that Azotobacter chroococcum bacteria synthesize antifungal anti-biotics, which gave it additional advantage for the use in field of production.Many efforts, however have been done in this respect, as Quoreshi (2003) who stated that inoculation with mycorrhizal fungi raised growth and nutrients uptake by Picea mariana seedlings. Likewise, Martin et al. (2003) mentioned that height and trunk diameter of Quercus palustris and $Q$. coccinea seedlings colonized with ectomycorrhizae were better that those of uncolonized ones. Abdel-Wahid et al. (2006) reported that using yeast twice at $4 \mathrm{~g} / \mathrm{L}+6 \mathrm{~g}$ NPK/plant significantly increased height, fresh and dry weights of shoots and N\% in roots of Euonymus japonicus plant, while yeast alone led to an increment in No. branches, stem diameter, root length, fresh and dry weights of roots, carotenoids content in the leaves and $\mathrm{K} \%$ in the roots. Sarhan et al. (2006) found that inoculation of vesicular arbuscular mycorrhize (VAM, Glomus sp.) at 100 or $200 \mathrm{ml} /$ pot significantly improved survival (\%), stem height and diameter and root length of Khaya senegalensis under salinity stress. Giri et al. (2007) postulated that mycorrhizal Acacia nilotica plants maintained greater root and shoot biomass than non-mycorrhizal ones. AM-inoculated plants had higher P, Zn, Mn and $\mathrm{Cu}$ concentrations than uninoculated plants. Similar observations were also revealed by Attia and Abdel-Azeem (2005) on Populus nigra, Sarhan et al. (2007) on jojoba, El-sayed et al. (2009) on Nephrolepis exaltata, Abdel-Fattah et al. (2009) on Dracaena and Ruscus and Abdel-Fattah et al., (2009) who pointed out that spraying the aqueous solution of active dry yeast $(8 \mathrm{~g} / \mathrm{L})$ greatly improved plant height, stem diameter, leaf No./plant, fresh and dry weights of aerial parts, as well as content of pigments, $\mathrm{N}, \mathrm{P}, \mathrm{K}, \mathrm{Fe}, \mathrm{Zn}$ and $\mathrm{Mn}$ in the leaves of Brassaia actinophylla plants.

The objective of this work is to detect the effect of some commercial biofertilizers on growth and active constituents of Narrow leaf fig transplants in comparison with NPK chemical fertilizer.

\section{MATERIALS AND METHODS}

Two pot experiments were performed in the open field at the nursery of Antoniades Research Branch, Hort. Res. Inst., Alexandria, Egypt during the two consecutive seasons of 2009 and 2010 to fined out the role of some commercial biofertilizers on improving growth and quality of Narrow leaf fig (Amstel Queen) plants in order to use rather than NPK chemical fertilizer. Therefore, six-months-old transplants of Ficus binnendykii (Miq.) Miq. cv. "Amstel Queen" of about $20 \mathrm{~cm}$ height and carrying about 8-10 leaves were planted on March, $1^{\text {st }}$ for the two seasons in 20-cm-diameter clay pots (one transplant/pot) filled with about $2.5 \mathrm{~kg}$ of an equal mixture of sand and clay (1:1, by volume). The physical and chemical properties of the soil mixture used in the two seasons were determined according to the standared methods described by Richards (1954) and averaged in Table (1). 
The transplants were kept to grow under full sun for one month (till April, $1^{\text {st }}$ ), as they were received the following treatments, which added as a soil dressing, five times with one month interval:

1-No fertilization, reffered to as control.

2-Chemical fertilization with a mixture of NPK (2:1:1) at the rate of $2 \mathrm{~g} /$ pot. Ammonium sulfate $(20.5 \%)$, calcium superphosphate $\left(15.5 \% \quad \mathrm{P}_{2} \mathrm{O}_{5}\right)$ and potassium sulfate $\left(48.5 \% \mathrm{~K}_{2} \mathrm{O}\right)$ fertilizers were used to obtain the required ratio.

3-Biofertilization with either Rhizobacterien (a commercial product contains a specific strain of Rhizobium sp.bacteria, conc. $10^{7}-10^{9}$ cells $/ \mathrm{ml}$ ), or Nitrobien (a commercial product contains a specific strain of Azospirillum barasilense bacteria, conc. $10^{7}-10^{9}$ cells $/ \mathrm{ml}$ ), or Biogien (a commercial product contains a specific clone of Azotobacter chroococcum bacteria, conc. $10^{6}$ cells $/ \mathrm{ml}$ ) at the rates of 3 and $6 \mathrm{~g} /$ pot for each.

Table (1): Some physical and chemical properties of the soil mixture used in 2009 and 2010 seasons

\begin{tabular}{|c|c|c|c|c|c|c|c|c|c|c|c|c|c|c|c|}
\hline \multirow{2}{*}{$\begin{array}{l}\tilde{\Xi} \\
\text { "ू } \\
\varpi \\
\tilde{D}\end{array}$} & \multicolumn{5}{|c|}{ Particle size distribution (\%) } & \multirow{2}{*}{ S.P. } & \multirow{2}{*}{$\begin{array}{l}\text { E.C. } \\
(\mathbf{d S} / \mathbf{m})\end{array}$} & \multirow{2}{*}{ pH } & \multicolumn{4}{|c|}{ Cations (meq/L) } & \multicolumn{3}{|c|}{ Anions (meq/L) } \\
\hline & $\begin{array}{l}\text { Coars } \\
\text { e sand }\end{array}$ & $\begin{array}{l}\text { Fine } \\
\text { sand }\end{array}$ & Silt & Clay & O.M & & & & $\mathrm{Ca}^{++}$ & $\mathbf{M g}^{++}$ & $\mathbf{N a}^{+}$ & $\mathbf{K}^{+}$ & $\mathrm{HCO}_{3}$ & $\mathrm{Cl}^{-}$ & $\mathrm{SO}_{4}^{--}$ \\
\hline 2009 & 15.90 & 26.20 & 19.10 & 37.10 & 1.70 & 45.65 & 2.99 & 7.85 & 18.21 & 9.33 & 31.50 & 1.42 & 3.67 & 26.10 & 30.69 \\
\hline 2010 & 18.76 & 23.88 & 21.33 & 34.46 & 1.57 & 43.79 & 3.36 & 7.90 & 21.73 & 10.26 & 33.11 & 1.53 & 3.89 & 24.89 & 37.85 \\
\hline
\end{tabular}

O.M.: Organic matter.

The layout of the experiments in the two seasons was a complete randomized design (Das and Giri, 1986) with three replicates and each replicate contained five transplants. The regular agricultural practices recommended for such plantation were carried out whenever needed. At the end of each season (on October, $30^{\text {th }}$ ), data were recorded as follows: plant height $(\mathrm{cm})$, stem diameter at the base $(\mathrm{mm})$, number of branches and leaves/plant, leaf area $\left(\mathrm{cm}^{2}\right)$, as well as fresh and dry weights of leaves, stem and roots $(\mathrm{g})$. In fresh leaf samples taken from the middle parts of the plants, photosynthetic pigments (chlorophyll a, b and carotenoids, $\mathrm{mg} / \mathrm{g}$ F.W.) were determined according to Moran (1982), while in dry ones, the percentages of total carbohydrates (Herbert et al., 1971), nitrogen using microKjeldahle method as described by Pregl (1945), phosphorus colorimetrically as the method indicated by Luatanab and Olsen (1965) and potassium using flamephotometer set (Jackson, 1973) were measured. Data were then tabulated and statistically analyzed according to SAS program (1994) using Duncan's Multiple Range Test (1955) for elucidating the significancy level between the means of the different treatments.

\section{RESULTS AND DISCUSSION}

Effect of fertilization treatments on: 1. Vegetative growth:Data in Table (2) show that all fertilization treatments employed in this study improved all vegetative growth parameters; expressed as: plant height $(\mathrm{cm})$, stem diameter $(\mathrm{mm})$, No. branches and leaves/plant and leaf area $\left(\mathrm{cm}^{2}\right)$ with various significance levels in the 
two seasons, but the greatest improvement in all of these parameters was due to biogien treatment at $6 \mathrm{~g} / \mathrm{pot}$, which gave the utmost high means in both seasons compared to control, NPK and other biofertilization treatments. However, rhizobacterien at $6 \mathrm{~g} /$ pot and biogien at $3 \mathrm{~g} /$ pot treatments improved some vegetative growth traits, especially in the second season giving values closely near to those gained by the prevalent treatment ( $6 \mathrm{~g}$ biogien/pot) with non-significant differences in some cases of the two seasons.

Table (2): Effect of fertilization treatments on some vegetative growth parameters of Ficus binnendykii (Miq) Miq. "Amstel Queen" transplants during 2009 and 2010 seasons.

\begin{tabular}{|l|c|c|c|c|c|}
\hline \multicolumn{1}{|c|}{ Treatments } & $\begin{array}{c}\text { Plant } \\
\text { height } \\
(\mathrm{cm})\end{array}$ & $\begin{array}{c}\text { Stem } \\
\text { diameter } \\
(\mathrm{mm} \text {. })\end{array}$ & $\begin{array}{c}\text { No. } \\
\text { branches } \\
\text { per plant }\end{array}$ & $\begin{array}{c}\text { No. } \\
\text { leaves } \\
\text { per plant }\end{array}$ & $\begin{array}{c}\text { Leaf } \\
\text { area } \\
\left(\mathrm{cm}^{2}\right.\end{array}$ \\
\hline & \multicolumn{5}{|c|}{ First season: 2009} \\
\hline Control & $37.52 \mathrm{e}$ & $3.21 \mathrm{~d}$ & $4.33 \mathrm{~d}$ & $23.36 \mathrm{e}$ & $16.30 \mathrm{e}$ \\
\hline NPK at 2g/pot & $45.00 \mathrm{c}$ & $3.85 \mathrm{dc}$ & $5.16 \mathrm{c}$ & $27.38 \mathrm{~d}$ & $19.51 \mathrm{c}$ \\
\hline Rhizobacterien at 3 g/pot & $42.46 \mathrm{dc}$ & $4.10 \mathrm{c}$ & $4.86 \mathrm{~cd}$ & $29.16 \mathrm{~cd}$ & $19.42 \mathrm{c}$ \\
\hline Rhizobacterien at 6 g/pot & $44.56 \mathrm{c}$ & $4.80 \mathrm{~b}$ & $4.98 \mathrm{~cd}$ & $34.10 \mathrm{ab}$ & $21.50 \mathrm{ab}$ \\
\hline Nitrobien at 3 g/pot & $39.72 \mathrm{ed}$ & $4.53 \mathrm{bc}$ & $5.13 \mathrm{c}$ & $25.95 \mathrm{ed}$ & $18.39 \mathrm{~d}$ \\
\hline Nitrobien at 6 g/pot & $41.33 \mathrm{~d}$ & $4.79 \mathrm{~b}$ & $5.46 \mathrm{cb}$ & $30.76 \mathrm{c}$ & $20.67 \mathrm{~b}$ \\
\hline Biogien at 3 g/pot & $49.28 \mathrm{~b}$ & $5.42 \mathrm{ab}$ & $5.81 \mathrm{~b}$ & $33.00 \mathrm{~b}$ & $20.58 \mathrm{~b}$ \\
\hline Biogien at 6 g/pot & $52.60 \mathrm{a}$ & $6.60 \mathrm{a}$ & $6.33 \mathrm{a}$ & $36.21 \mathrm{a}$ & $22.33 \mathrm{a}$ \\
\hline & \multicolumn{5}{|c|}{$\mathrm{Second} \mathrm{season:2010}$} \\
\hline Control & $35.90 \mathrm{e}$ & $3.40 \mathrm{~d}$ & $4.40 \mathrm{~d}$ & $26.51 \mathrm{e}$ & $15.06 \mathrm{e}$ \\
\hline NPK at 2g/pot & $43.10 \mathrm{c}$ & $4.33 \mathrm{~cd}$ & $5.31 \mathrm{c}$ & $31.83 \mathrm{~cd}$ & $18.00 \mathrm{c}$ \\
\hline Rhizobacterien at 3 g/pot & $43.81 \mathrm{c}$ & $4.68 \mathrm{c}$ & $5.00 \mathrm{~cd}$ & $33.10 \mathrm{c}$ & $17.85 \mathrm{c}$ \\
\hline Rhizobacterien at 6 g/pot & $46.35 \mathrm{ab}$ & $5.31 \mathrm{~b}$ & $5.19 \mathrm{~cd}$ & $37.81 \mathrm{a}$ & $19.80 \mathrm{ab}$ \\
\hline Nitrobien at 3 g/pot & $40.23 \mathrm{~d}$ & $5.00 \mathrm{~b}$ & $5.33 \mathrm{c}$ & $30.78 \mathrm{~d}$ & $17.00 \mathrm{~d}$ \\
\hline Nitrobien at 6 g/pot & $45.20 \mathrm{~b}$ & $5.71 \mathrm{ba}$ & $5.67 \mathrm{cb}$ & $35.56 \mathrm{~b}$ & $19.03 \mathrm{~b}$ \\
\hline Biogien at 3 g/pot & $46.18 \mathrm{~b}$ & $6.03 \mathrm{ab}$ & $5.90 \mathrm{~b}$ & $36.23 \mathrm{ba}$ & $18.36 \mathrm{bc}$ \\
\hline Biogien at 6 g/pot & $48.79 \mathrm{a}$ & $6.92 \mathrm{a}$ & $6.40 \mathrm{a}$ & $38.25 \mathrm{a}$ & $20.76 \mathrm{a}$ \\
\hline
\end{tabular}

Means within a column having the same letters are not significantly different according to Duncan's Multiple Range Test (DMRT) at 5\% level.

A similar trend was also obtained concerning the fresh and dry weights (g) of leaves, stem and roots (Table, 3 ), as they were significantly increased in response to applying either NPK or biofertilizers used in this work, with the superiority of 6 $\mathrm{g} /$ pot biogien treatment, which scored the higheat records in the two seasons and followed by the treatment of biogien at $3 \mathrm{~g} /$ pot that gave some values in the same rank of the super-treatment. This may be attributed to the capability of biogien in fixing more atmospheric $\mathrm{N}$ and secrete more vitamins and growth promoting substances necessary for good and healthy growth (Darwish, 2002).

In addition, Subba Rao (1993) affirmed that Azotobacter chroococcum (the bacteria found in biogien) synthesize antifungal antibiotics which gave it a special advantage for the use in field of production. These results, however are coincidenced 
with those attained by Martin et al. (2003) on Quercus palustris, Sarhan et al. (2006) on Khaya senegalensis, Giri et al. (2007) on Acacia nilotica and AbdelFattah et al. (2009) on Dracaena and Ruscus.

Table (3): Effect of fertilization treatments on fresh and dry weights of Ficus binnendykii (Miq) Miq. "Amstel Queen" leaves, stem and roots during 2009 and 2010 seasons.

\begin{tabular}{|l|c|c|c|c|c|c|c|}
\hline \multirow{2}{*}{ Treatments } & \multicolumn{3}{|c|}{ Fresh weight $(\mathrm{g})$} & \multicolumn{3}{c|}{ Dry weight (g) } \\
\cline { 2 - 7 } & Leaves & Stem & Roots & Leaves & Stem & Roots \\
\hline & \multicolumn{5}{|c|}{ First season: 2009} \\
\hline Control & $8.82 \mathrm{f}$ & $10.00 \mathrm{e}$ & $10.98 \mathrm{~g}$ & $1.27 \mathrm{e}$ & $3.50 \mathrm{~d}$ & $4.50 \mathrm{~g}$ \\
\hline NPK at 2g/pot & $10.56 \mathrm{fe}$ & $12.33 \mathrm{~d}$ & $13.21 \mathrm{ef}$ & $1.53 \mathrm{~d}$ & $4.21 \mathrm{c}$ & $5.33 \mathrm{f}$ \\
\hline Rhizobacterien at 3 g/pot & $13.13 \mathrm{~d}$ & $13.78 \mathrm{~cd}$ & $16.28 \mathrm{~d}$ & $2.71 \mathrm{c}$ & $4.81 \mathrm{cb}$ & $7.26 \mathrm{~d}$ \\
\hline Rhizobacterien at 6 g/pot & $14.83 \mathrm{c}$ & $14.50 \mathrm{c}$ & $18.50 \mathrm{c}$ & $3.53 \mathrm{~b}$ & $5.03 \mathrm{~b}$ & $8.33 \mathrm{c}$ \\
\hline Nitrobien at 3 g/pot & $11.57 \mathrm{e}$ & $11.90 \mathrm{de}$ & $13.10 \mathrm{fe}$ & $2.50 \mathrm{~cd}$ & $4.20 \mathrm{c}$ & $5.94 \mathrm{ef}$ \\
\hline Nitrobien at 6 g/pot & $13.10 \mathrm{~d}$ & $13.87 \mathrm{~cd}$ & $14.38 \mathrm{e}$ & $2.87 \mathrm{c}$ & $4.83 \mathrm{cb}$ & $6.43 \mathrm{e}$ \\
\hline Biogien at 3 g/pot & $17.54 \mathrm{~b}$ & $16.33 \mathrm{~b}$ & $20.33 \mathrm{~b}$ & $3.96 \mathrm{~b}$ & $5.71 \mathrm{ba}$ & $9.13 \mathrm{~b}$ \\
\hline Biogien at 6 g/pot & $20.71 \mathrm{a}$ & $18.56 \mathrm{a}$ & $21.78 \mathrm{a}$ & $5.00 \mathrm{a}$ & $6.30 \mathrm{a}$ & $9.80 \mathrm{a}$ \\
\hline & \multicolumn{5}{|c|}{ Second season:2010 } \\
\hline Control & $10.00 \mathrm{f}$ & $10.53 \mathrm{e}$ & $11.73 \mathrm{f}$ & $2.15 \mathrm{e}$ & $3.61 \mathrm{~d}$ & $5.00 \mathrm{f}$ \\
\hline NPK at 2g/pot & $11.93 \mathrm{ef}$ & $12.70 \mathrm{de}$ & $14.10 \mathrm{ef}$ & $2.60 \mathrm{~d}$ & $4.32 \mathrm{~cd}$ & $6.20 \mathrm{e}$ \\
\hline Rhizobacterien at 3 g/pot & $14.16 \mathrm{~d}$ & $14.68 \mathrm{c}$ & $17.80 \mathrm{c}$ & $2.95 \mathrm{~cd}$ & $5.11 \mathrm{cb}$ & $8.01 \mathrm{c}$ \\
\hline Rhizobacterien at 6 g/pot & $15.60 \mathrm{c}$ & $16.00 \mathrm{~b}$ & $20.35 \mathrm{~b}$ & $3.71 \mathrm{c}$ & $5.58 \mathrm{~b}$ & $9.00 \mathrm{bc}$ \\
\hline Nitrobien at 3 g/pot & $12.35 \mathrm{e}$ & $13.50 \mathrm{~d}$ & $14.50 \mathrm{e}$ & $2.30 \mathrm{de}$ & $4.73 \mathrm{c}$ & $6.53 \mathrm{de}$ \\
\hline Nitrobien at 6 g/pot & $13.78 \mathrm{~d}$ & $16.20 \mathrm{~b}$ & $16.19 \mathrm{~d}$ & $3.23 \mathrm{c}$ & $5.63 \mathrm{~b}$ & $7.29 \mathrm{~d}$ \\
\hline Biogien at 3 g/pot & $18.10 \mathrm{~b}$ & $18.61 \mathrm{a}$ & $20.70 \mathrm{~b}$ & $4.47 \mathrm{~b}$ & $6.50 \mathrm{a}$ & $9.32 \mathrm{~b}$ \\
\hline Biogien at 6 g/pot & $21.85 \mathrm{a}$ & $18.93 \mathrm{a}$ & $22.26 \mathrm{a}$ & $5.32 \mathrm{a}$ & $6.61 \mathrm{a}$ & $10.03 \mathrm{a}$ \\
\hline
\end{tabular}

Means within a column having the same letters are not significantly different according to Duncan's Multiple Range Test (DMRT) at 5\% level.

2. Chemical composition:From data averaged in Table (4), it could be concluded that dressing with biogien at either 3 or $6 \mathrm{~g} /$ pot gave the pronounced beneficial effect on active constituents of the fertilized plants with few exceptions in the two seasons. However, the mastery in all measured constituents was for the high rate of biogien $(6 \mathrm{~g} / \mathrm{pot})$, which gave more green leaves and higher percentages of total carbohydrates, N, P and K when compared to control, NPK and other biofertilization treatments. Application of rhizobacterien, especially at $6 \mathrm{~g} / \mathrm{pot}$ improved the content of $\mathrm{P}$ and $\mathrm{K}$ to reach the same significance level of dressing with biogien at $6 \mathrm{~g} / \mathrm{pot}$. This may indicate the role of biofertilizers in fixing atmospheric $\mathrm{N}$ and mobilizing phosphate and other nutrients to be available for plants (Quoreshi, 2003). Analogus observations were also noticed by Attia and Abdel-Azeem (2005) on Populus nigra, Sarhan et al. (2007) on jojoba, El-Sayed et al. (2009) on Nephrolepis exaltata and Abdel Fattah et al. (2009) on Brassaia actinophylla. According to the afforestated results, it could be recommended to fertilize the 6-months-old transplants of Ficus binnendykii cv. "Amstel Queen" grown in $20-\mathrm{cm}$-diameter clay pots filled with about $2.5 \mathrm{~kg}$ of sand + clay mixture $(1: 1, \mathrm{v} / \mathrm{v})$ with biogien biofertilizer at the rate of $6 \mathrm{~g} / \mathrm{pot}$, five times (commencing from April, ${ }^{\text {st }}$ ) with one month interval to score the best growth and high quality. 
Table (4): Effect of fertilization treatments on chemical composition of Ficus binnendykii (Miq) Miq. "Amstel Queen" leaves during 2009 and 2010 seasons

\begin{tabular}{|c|c|c|c|c|c|c|c|}
\hline \multirow[b]{2}{*}{ Treatments } & \multicolumn{3}{|c|}{ Pigments content (mg/g F.W.) } & \multirow[b]{2}{*}{$\begin{array}{l}\text { Total carbohydrates } \\
(\%)\end{array}$} & \multirow[b]{2}{*}{$\mathbf{N}(\%)$} & \multirow[b]{2}{*}{$\mathbf{P}(\%)$} & \multirow[b]{2}{*}{$\mathbf{K}(\%)$} \\
\hline & \begin{tabular}{|c|} 
Chlorophyll \\
a
\end{tabular} & $\begin{array}{c}\text { Chlorophyl } \\
\text { I b }\end{array}$ & Carotenoids & & & & \\
\hline & \multicolumn{7}{|c|}{ First season: 2009} \\
\hline Control & $0.90 \mathrm{e}$ & $0.21 \mathrm{e}$ & $0.63 \mathrm{f}$ & $27.98 \mathrm{f}$ & $1.20 \mathrm{f}$ & $0.09 \mathrm{~d}$ & $1.10 \mathrm{~d}$ \\
\hline NPK at 2g/pot & $1.40 \mathrm{c}$ & $0.38 \mathrm{~cd}$ & $0.78 \mathrm{e}$ & $33.21 \mathrm{e}$ & $1.37 \mathrm{e}$ & $0.11 \mathrm{~d}$ & $1.38 \mathrm{c}$ \\
\hline Rhizobacterien at 3 g/pot & $1.06 \mathrm{~d}$ & $0.40 \mathrm{c}$ & $0.89 \mathrm{~d}$ & $41.76 \mathrm{~d}$ & $1.85 \mathrm{c}$ & 0.21 b & $1.56 \mathrm{~b}$ \\
\hline Rhizobacterien at 6 g/pot & $1.47 \mathrm{c}$ & $0.53 \mathrm{~b}$ & $1.00 \mathrm{c}$ & $48.90 \mathrm{c}$ & $1.94 \mathrm{~b}$ & 0.25 a & $1.77 \mathrm{ab}$ \\
\hline Nitrobien at 3 g/pot & $1.07 \mathrm{~d}$ & $0.30 \mathrm{~d}$ & $0.90 \mathrm{~d}$ & $41.40 \mathrm{~d}$ & $1.47 \mathrm{e}$ & $0.16 \mathrm{c}$ & $1.21 \mathrm{~d}$ \\
\hline Nitrobien at 6 g/pot & $1.33 \mathrm{~cd}$ & $0.35 \mathrm{~cd}$ & $1.07 \mathrm{c}$ & 50.33 c & $1.63 \mathrm{~d}$ & $0.18 \mathrm{cb}$ & $1.38 \mathrm{c}$ \\
\hline Biogien at $3 \mathrm{~g} / \mathrm{pot}$ & $1.80 \mathrm{~b}$ & 0.58 b & $1.30 \mathrm{~b}$ & $54.60 \mathrm{~b}$ & $2.10 \mathrm{~b}$ & 0.23 a & $1.60 \mathrm{~b}$ \\
\hline \multirow[t]{2}{*}{ Biogien at $6 \mathrm{~g} / \mathrm{pot}$} & $2.25 \mathrm{a}$ & 0.95 a & $1.67 \mathrm{a}$ & $60.91 \mathrm{a}$ & $2.33 \mathrm{a}$ & 0.28 a & $1.93 \mathrm{a}$ \\
\hline & \multicolumn{7}{|c|}{ Second season:2010 } \\
\hline Control & $1.03 \mathrm{e}$ & $0.33 \mathrm{f}$ & $0.71 \mathrm{f}$ & $28.20 \mathrm{f}$ & $1.16 \mathrm{~g}$ & $0.11 \mathrm{c}$ & $1.05 \mathrm{e}$ \\
\hline NPK at 2g/pot & $1.51 \mathrm{~d}$ & $0.48 \mathrm{e}$ & $0.88 \mathrm{e}$ & $36.28 \mathrm{e}$ & $1.24 \mathrm{f}$ & 0.15 bc & $1.31 \mathrm{~d}$ \\
\hline Rhizobacterien at $3 \mathrm{~g} /$ pot & $1.65 \mathrm{~cd}$ & $0.64 \mathrm{~d}$ & $1.00 \mathrm{~d}$ & $45.87 \mathrm{~d}$ & $1.72 \mathrm{~d}$ & $0.24 \mathrm{a}$ & $1.50 \mathrm{c}$ \\
\hline Rhizobacterien at 6 g/pot & $1.75 \mathrm{c}$ & $0.87 \mathrm{c}$ & $1.16 \mathrm{c}$ & $52.10 \mathrm{c}$ & $1.91 \mathrm{c}$ & $0.30 \mathrm{a}$ & $1.69 \mathrm{ab}$ \\
\hline Nitrobien at 3 g/pot & $1.58 \mathrm{~cd}$ & $0.65 \mathrm{~d}$ & $1.03 \mathrm{~d}$ & $45.00 \mathrm{~d}$ & $1.50 \mathrm{e}$ & 0.15 bc & $1.23 \mathrm{~d}$ \\
\hline Nitrobien at $6 \mathrm{~g} /$ pot & $1.97 \mathrm{~b}$ & $0.80 \mathrm{c}$ & $1.21 \mathrm{c}$ & 53.87 bc & $1.68 \mathrm{~d}$ & 0.18 b & $1.40 \mathrm{c}$ \\
\hline Biogien at $3 \mathrm{~g} / \mathrm{pot}$ & $2.10 \mathrm{~b}$ & $1.06 \mathrm{~b}$ & $1.46 \mathrm{~b}$ & 55.86 b & $2.13 \mathrm{~b}$ & $0.25 \mathrm{a}$ & $1.62 \mathrm{~b}$ \\
\hline Biogien at $6 \mathrm{~g} / \mathrm{pot}$ & $2.59 \mathrm{a}$ & $1.25 \mathrm{a}$ & $1.81 \mathrm{a}$ & $64.61 \mathrm{a}$ & $2.70 \mathrm{a}$ & $0.29 \mathrm{a}$ & $1.89 \mathrm{a}$ \\
\hline
\end{tabular}

Means within a column having the same letters are not significantly different according to Duncan's Multiple Range Test (DMRT) at 5\% level.

\section{إستجابة شتّلات فيكس (أمستيل كوين) لبعض الأسمدة الحيوية

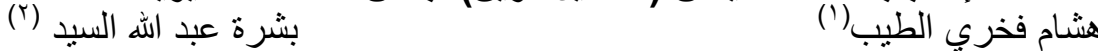

( (1) قسم بحوث الحدائق النباتية، معهد بحوث البساتين، مركز البحوث الزر اعية، حديقة أنطونياد،

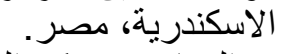

( r) قسم بحوث الزينة، معهد بحوث البساتين، مركز البحوث الزراعية، الجيزة، مصر.

\section{الخلاصة}

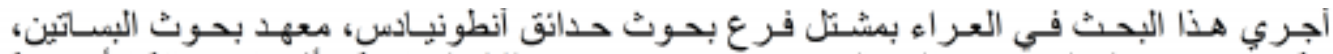

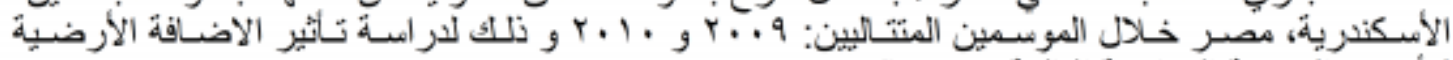

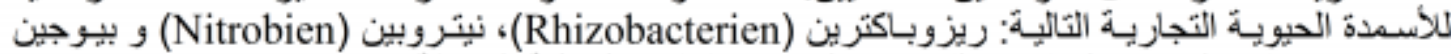

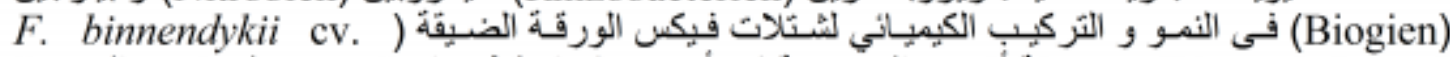

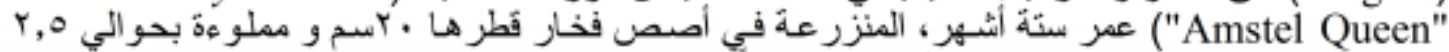

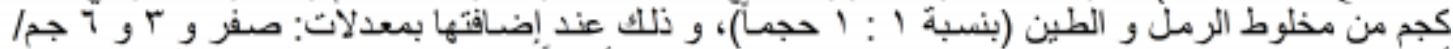

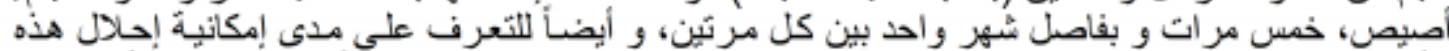

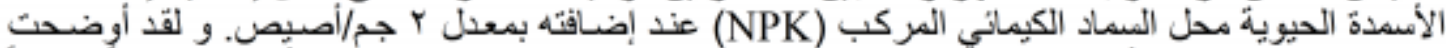

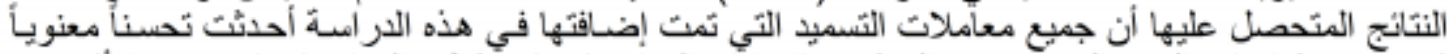

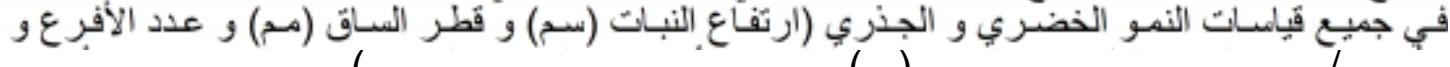

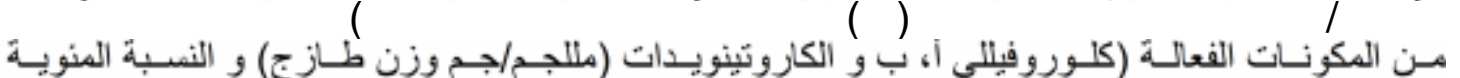

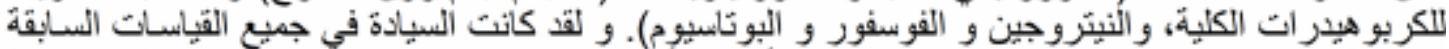

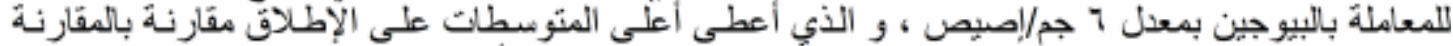

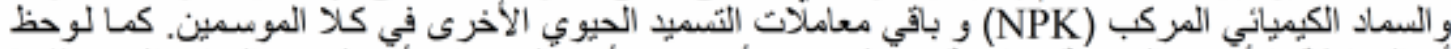

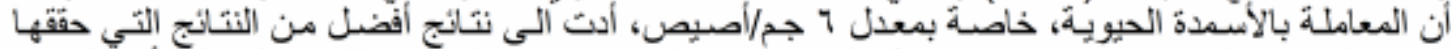

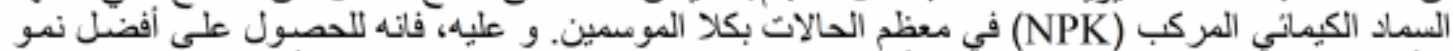

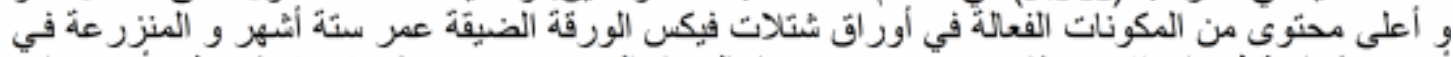

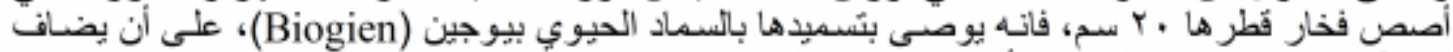
|أصيص و بفاصل شهر واحد بين كل مرنين.

\section{REFERENCES}

Abdel-Fattah, H. Gehan; A. El-Sayed Boshra and A. M. Khenizy Soad (2009). Response of Dracaena and Ruscus plants to humic acid and biofertilizer supply. Annals of Agric. Sci., Moshtohor, 47(1): 111-119. 
Abdel-Fattah, H. Gehan; M. Abdel-Moniem Azza and M.A.I. El-Shamy (2009). Response of Australian umbrella tree (Brassaia) transplants to some fertilization, salysilic acid and dry yeast treatments. Annals of Agric. Sci., Moshtohor, 47(2):275-283.

Abdel-Wahid, M. K. Safwat; Y. Labib Naglaa and B.B. Rezk-Alla (2006). Effect of active dry yeast and chemical fertilization on vegetative growth and the main constituents of Euonymus japonicus Thunb. plant. Fayoum J. Agric. Res. \& Dev., 20(1):136-147.

Attia, Elham M. and H.M. Abdel-Azeem Hoda (2005). Effect of biofertilization on yield and active materials in Lawsonia inermis L. plants. The $6^{\text {th }}$ Arab. Conf. Hort., March 20-22, Dept. Hort., Fac. Agric., Ismailia, Egypt:109-128.

Darwish, F.M. (2002). Effect of different fertilizer sources and levels on growth, yield and quality of tomato. Ph. D. Thesis, Fac. Agric., Cairo univ., Egypt.

Das, M.N. and N.C. Giri (1986). Design and Analysis of Experiments. $2^{\text {nd }}$ Ed., Published by Mohinder Singh Sejwal for Wiley, New Delhi 110 002, 488 pp.

Duncan, D.B. (1955). Multiple range and multiple F-tests. J. Biometrics, 11:1-42.

El-Sayed, A. Boshra; A. El-Din Hanan and S.M.Shahin (2009). Response of Nephrolepis exaltata Schatt. plant to medium type and nitrobien biofertilization. J. Bio. Chem. Environ. Sci., 4(1):705-717.

Giri, B.; R. Kapoor and K. G. Mukerji (2007). Improved tolerance of Acacia nilotica to salt stress. Microbial Ecology, 54 (4):753-760.

Herbert, D.; P.J. Philips and R.E. Strange (1971). Determination of total carbohydrates. Methods in Microbiology, 5(8):290-344.

Huxley, A.; M. Griffiths and M. Levy (1992). The New Royal Hort. Society Dictionary of Gardening. The Stockton Press, 257 Park Avenue South, New York, NY 10010, USA, vol. 2, $747 \mathrm{pp}$.

Jackson, M.L. (1973). Soil Chemical Analysis. Prentice-Hall of India Private Limited M97, New Delhi, India, 498 pp.

Luatanab, F.S. and S.R. Olsen (1965). Test of an ascorbic acid method for determining phosphorus in water and $\mathrm{NaHCO}_{3}$ extracts from soil. Soil Sci. Soc. Amer. Proc., 29:677-678.

Martin, T.P.; J.R. Harris; G.K. Eaton and O.K. Miller (2003). The efficacy of ectomycorrhizal colonization of pin and scarlet oak in nursery production. J. Environ. Hort. 21(1):45-50.

Moran, R. (1982). Formula for determination of pigments extracted with N, N-dimethyl formamide. Plant Physol., 69:1376-1381.

Pregl, F. (1945). Quantitative Organic Micro-Analysis, $4^{\text {th }}$ Ed., J. and A. Churchill Ltd., London, p:203-209.

Quoreshi, A.M. (2003). Nutritional preconditioning and ectomycorrhizal formation of Picea mariana (Mill.) B.S.P. seedlings. Eurasian J. Forests Res., 6:1-63.

Richards, L.A. (1954). Diagnosis and Improvement of Saline and Alkali Soils. USDA, Handbook No. 60.

Sarhan, A.Z.; Effat I. El-Maadawy and S.S. Surour (2007). Effect of biopromotors and biofertilizers application on growth and chemical somposition of jojoba plants. J. Agric. Sci., Mansoura Univ., 32(8):6575-6598.

Sarhan, A.Z ; M.A. Salem and L. Soliman (2006). Effect of irrigation water salinity levels and soil mycorrhizal fungi inoculation on growth of African mahogany (Khaya senegalensis) transplants. Ann. Agric. Sci., Moshtohor, 44(2):625-635.

SAS Institute (1994). SAS/STAT User's Guide: Statistics. Vers. 6.04, $4^{\text {th }}$ Ed., SAS Institute Inc., Cary, N.C., USA.

Subba Rao, N.S. (1993). Biofertilizers in Agriculture, $3^{\text {rd }}$ Ed., Oxford \& IBH Publishing Co. Ltd., New Delhi, Bombay, Calcutta, 242 p. 